190. ECHINOPEPON RACEMOSUS (STEUD.) C. JEFFREY. A NEW RECORD OF CUCURBITACEAE FOR THE FLORA OF SOUTHERN BRAZIL

Luís Fernando PAIVA LIMA

Recibido el 13 de mayo de 2008, aceptado para su publicación el 8 de julio de 2009 Publicado "on line" en julio de 2009

Echinopepon racemosus (Steud.) C. Jeffrey. Una nueva cita de Cucurbitaceae para la flora del sur del Brasil.

Palabras clave. Cucurbitaceae, Echinopepon racemosus, corologia, sur del Brasil.

Key words. Cucurbitaceae, Echinopepon racemosus, corology, southern Brazil

The knowledge about Cucurbitaceae family from southern Brazil is still scarce. The most relevant work is a floristic study by Porto (1974) in Rio Grande do Sul State. However, in South America, the species number of this family decreases gradually as the latitude increases thus Apodanthera sagittifolia (Griseb.) Mart. Crov. is the most southern species known (Martinez-Crovetto, 1946).

The genus Echinopepon Naud. can be easily recognized due to its monoics, small whitish flowers, where the estaminates are gathered in racemes or panicules and the pistilates are solitary or in small fascicules, coaxillary with the estaminates, as well as due to fibrous spiny pyxidium with a smooth apical operculum.

This genus that belongs to the tribe Sicyeae Scharad., subtribe Cyclantherinae C. Jeffrey (Jeffrey, 2005), includes 18 New World taxa whose center of diversity at is the Pacific coast of Mexico from middle to high elevation montains (Jeffrey, 2005; Monro \& Stafford, 1998). Only two species are found in South America: E. racemosus, whith southern limit of distribution at the Salta Province in Argentina, and E. disjunctus Pozner, whose type material comes from Jujuy Province in Argentina (Pozner, 2004). About its ecology, these species prefer the heliophilous and perturbed areas of tropical forests, coniferous forests, agricultural areas, as well as road sidelines and streams (Rodriguez, 1995).

The municipality of Urubici, in Santa Catarina State, represents the southern limit in the South America. The new location stands over two degrees south latitude far from the southern limit informed by Monro \& Stafford (1998), therefore this data represents an extension of the known limits for this species.

\section{Specimens examined}

BRAZIL. SANTA CATARINA. Urubici, margem do Rio Canoas, 18/01/1960, J. Mattos 7508 (HAS, PEL). Serra da Goiabeira, 12/04/1982, J. Mattos 23404 (HAS). Próximo ao Rio Rufino, 23/03/1983, J. Mattos 24207 (HAS).

\section{BIBLIOGRAPHY}

RODRIGUEZ, J. C. -1995- Distribución geográfica del gênero Equinopepon (Cucurbitaceae). Anales Inst. Biol. Univ. Nac. Auton. México, Bot. 66(2): 171-181.

JEFFREY,C. -2005-A new system of Cucurbitaceae. Bot. Zhurn. 90: 332-335. MARTINEZCROVETTO, R. -1946- Nota taxonômica sobre 
Wilbrandia sagittifolia Griseb. (Cucurbitaceae). Bol. Soc. Argent. Bot. 1(4): 312-317.

MONRO,A. K. \& P. J. STAFFORD, -1998-Asynopsis of the genus Echinopepon (Cucurbitaceae; Sicyeae), including three new taxa. Ann. Missouri Bot. Gard. 85: 257-272.

PORTO, M. L. -1974- Cucurbitaceae. In: A. R. Schultz, ed., Flora Ilustrada do Rio Grande do Sul. Fasc. VIII. Univ. Fed. Rio Grande do Sul, Porto Alegre.
POZNER, R. -2004- A new species of Echinopepon from Argentina and taxonomic notes on the subtribe Cyclantherinae (Cucurbitaceae). Syst. Bot. 29(3): 599-608.

Dirección del autor. Instituto de Biociências, Av. Bento Gonçalves, 9500 - Bloco IV - Prédio 43433 - sala 214, Universidade Federal do Rio Grande do Sul. Brasil. luislima@redemeta.com.br

191. NUEVO HÍBRIDO DEL GÉNERO THYMUS (LABIATAE)

Ramón MORALES

Recibido el 6 de junio de 2009, aceptado para su publicación el 8 de junio de 2009 Publicado "on line" en junio de 2009

A new hybrid in the genus Thymus (Labiatae)

Palabras clave. Thymus $x$ viteki, Th. vulgaris, Th. fontqueri, Lérida.

Key words. Thymus $x$ viteki, Th. vulgaris, Th. fontqueri, Lérida.

Thymus $x$ viteki $\mathrm{R}$. Morales hybr. nov. Thymus fontqueri (Jalas) Molero \& Rovira $\mathrm{x}$ Th. vulgaris L. subsp. vulgaris

A Th. vulgare differt calycibus pilosis, dentibus superioribus ciliatis; a Th. fontqueri habitu procumbenti accedit, floribus brevioribus recedit.

Holotypus: "Spanien; Catalunya, SW von Tremp, Sierra de Montsec, Weg von Alsamora zur Congost de Mont-rebei; $866 \mathrm{~m}$; 42 04 ' 53'N / 043'45"E, 25.05.2003, E. Vitek 03-0099a", MA 731986.

La localidad donde fue recolectada la planta se encuentra en la provincia de Lérida, casi en el límite con la de Huesca, muy cercana al río Noguera Ribagorzana, en la cuadrícula UTM 31TCG16. Según citas dadas por O. Bolós en el Atlas de los Países Catalanes 12, $\mathrm{n}^{\circ} 2951$ y 2975 , en dicha cuadrícula están presentes Thymus vulgaris L. subsp. vulgaris y Thymus fontqueri.

Presenta caracteres intermedios entre las especies progenitoras; de hábito desordenado, tendido o medio rastrero, con hojas grandes planas, apenas pelosas o con pelos cortos en el margen, y las pequeñas que nacen en fascículos, algunas de ellas muy pelosas por el envés como en Th. vulgaris. Inflorescencia laxa. 